\title{
A brief review on the structural analysis of Graphene Oxide.
}

\author{
Reshmi Bose ${ }^{a}$ \\ a Department of Basic Science and Humanities, \\ University of Engineering and Management (UEM), \\ B/5, Plot No.III, Action Area-III, Newtown, Kolkata 700156
}

(Dated:)

\begin{abstract}
Graphene is considered as one of the important allotropes of carbon as two dimension honeycomb lattice. Graphene can be oxidised into graphene oxide (GO) by several process and due to presence of oxygen containing functional groups on the basal plane graphene oxide is non conductive in nature but it can readily dissolved in most of the solvents like water. Thus GO can be used in different fields of material science like in batteries, capacitor and solar cells. This review article covers a brief review on the structural analysis of graphene oxide like the presence of oxygen containing functional groups and their positions, various spectral analysis of graphene oxide and other such studies.
\end{abstract}

PACS numbers:

\section{INTRODUCTION}

One of the important allotropes (carbon nanotube, fullerene, diamond) of elemental carbon is Graphene, with a planar monolayer of carbon atoms arranged into a two-dimensional (2D) honeycomb lattice with a carboncarbon bond length of $0.142 \mathrm{~nm}$ [1]. Electrons in graphene contribute to some special properties such as an anomalous quantum Hall effect and the absence of localization as they behave like mass less relativistic particles [2,3]. Graphene [2] shows a variety of quaint properties including high electron mobility at room temperature (250,000 cm2/Vs)[4,5] exceptional thermal conductivity (5000 W m1 K1) [6] and superior mechanical properties with Youngs modulus of $1 \mathrm{TPa}$ [7]. The study of the electronic properties of graphene and its chemical derivatives opens a new era of solid state electronics [8] and of composite materials [9]. The unique electronic and mechanical properties of graphene suggest various interesting applications, the requisite large-scale direct synthesis and solution-based handling have proven difficult $[10,11]$. The exfoliation of graphite oxide (GO) opens a new era to material science. The material first discovered by the Oxford chemist Benjamin Brodie in 1859 produces atomically thin graphene oxide sheets that are dispersible in basic media [12]. It is an electronically hybrid material that features both conducting -states from sp2 carbon sites and a large energy gap (carrier transport gap) between the -states of its sp3-bonded carbons [13]. Due to the peculiar insulating and defective nature of GO, it does not allow the observation of fundamental two-dimensional condensed-matter effects, which has limited the interest of physicists in the material. Although GO has given special interest by Chemists because of its heterogeneous chemical and electronic structures, along with the fact that it can be processed in solution [14]. This review article briefly discuss about the Structure of Graphene Oxide and Reduced Graphene Oxide and Electrical Conductivity of Individual Graphene Oxide Sheets Reduced at low Temperatures.

\section{STRUCTURE OF GRAPHENE OXIDE}

The structure of GO has found to be non stoichiometric in chemical composition with ranging from $\mathrm{C} 8 \mathrm{O} 2 \mathrm{H} 3$ to C8O4H5, considering C:O ratio of $4: 1$ to $2: 1$.[1518] The density functional theory (DFT) have indicated that it becomes extremely difficult to reduce GO with $\mathrm{C}: \mathrm{O}$ ratios above 16:1[19]. Some of the key questions arising to the structure of GO are: i) which oxygen containing functional groups are present? ii) What is their function? iii) Is it even possible to form graphene as a product of reduction of GO? The accurate answers of the above questions are still unknown but from the recent findings in the solid-state NMR characterization of 13C-labeled graphite oxide [20] and from the other works [21-23] it can be concluded that the Lerf-Klinowski [24] and the De ka ny models [25] can give satisfactory answers. The information obtained from the analytical studies are not satisfying to understand the whole concept of the optical and electrical properties of $\mathrm{GO} / \mathrm{rGO}$ because they depend on the distribution of the functional groups [26]. The functional groups available on the basal plane mainly consist of oxygen-containing functional groups like hydroxyls and epoxies whereas smaller amounts of carboxyl, carbonyl, phenol, lactone, and quinone are present primarily at the sheet edges [27]. The functional groups present at sheet edge interact with a variety of organic and inorganic materials in non-covalent, covalent and/or ionic manner. It helps to synthesis various unusual functional hybrids and composites which shows intriguing properties. GO is found to be a covalent material with $60 \%$ of carbon atoms in the basal plane. The carbon atoms present with in basal plane are sp3 hybridized due to presence of oxygen atoms in the form of epoxy and hydroxyl groups but an graphene sheet should be consist of only sp2 hybridized carbon atoms[24]. By contrast recent findings confirms that GO is a two dimensional sheet with both sp2 and sp3 carbon atoms. [14]. This non stoichiometric nature of GO is attribute to the presence of size,shape and sp3 and sp2 hybridised carbon atoms.[14] The optical and electrical

\section{American Journal of Physical Sciences and Applications US:ISSN 2644-0792 website:http://thesmartsociety.org/ajpsa/}


properties of GO depends on the sp2 hybridised Carbon atoms present.[28]. This kind of material is in generally amorphous in nature. It may contain poor or no translational symmetry [28]. Due to presence of sp3 hybridised oxygen containing functional groups the basal plane of GO is non uniform in nature where the small finite sp2 clusters confine within the sp3 hybridised C$\mathrm{O}$ matrix which can be explained by scanning tunnelling microscopy[29,30], Raman spectroscopy[31,32], transport studies $[33,34]$ and high resolution transmission electron microscopy $[35,36]$. The carbon atoms present on the GO can extent themselves and can stack with each other by forming layered structure like graphene [37]. The spacing between the layers depends on the humidity as GO is hygroscopic in nature it absorbs moisture from air thus changing in the interlayer distance from $0.6 \mathrm{~nm}$ to $0.12 \mathrm{~nm}$ for dry and hydrated structure [38]. Now during thermal reduction of GO this trapped water molecules and the some of the oxygen containing groups such as CO and $\mathrm{CO} 2$ comes out of the structure leading to the interlayer shrinkage $[39,40]$. Few defects such as double vacancy or 5-8-5 defects and StoneWales or 5775 defects arise due to removal of oxygen from the GO structure [41, 42].

\section{A. Raman spectroscopy}

Raman spectroscopy is the best way to determine the structural informations about Carbon based material [43]. In Graphene like material generally G and D peaks along with their overtones appears due to the vibration of $\mathrm{sp} 2$ carbons. The $\mathrm{G}$ peak appears due to the presence of E2g photons in Brillouin zone centre and D peak appears due to breathing of aromatic ring and only activated when there are some defects [44]. This D peaks are of weak intensities and broad that leads to the measurement of degree of disorder [45]. The G and D peaks appears at around $1580 \mathrm{~cm}^{-1}$ and $1350 \mathrm{~cm}^{-1}$ respectively. The $2 \mathrm{D}$ peak appears around $2680 \mathrm{~cm}^{-} 1$, are the overtones of $\mathrm{D}$ peaks. The shift and shape of the $2 \mathrm{D}$ peak depends on the number of graphene layers. The 2D peak can arise even without presence of any defects. A D $+\mathrm{D}$ peak also appeared due to defect activation [46]. During reduction of GO the interlayer species and oxygen containing functional groups are removed this results in stronger interlayer coupling between the adjacent layers thus blue shift is observed in $2 \mathrm{D}$ peak of multilayer GO [47].

\section{B. Fluorescence in GO}

Due to the heterogeneous electronic and atomic structure fluorescence is possible nearly at near-infrared (NIR), visible and ultraviolet fluorescence region [4855]. The fluorescence in GO is attribute to the electron-hole recombination in local electronic states arising from different configurations. As in graphene the finite sized sp2 clusters are present within the sp3 matrix this tie up the - electrons in GO and give rise to electron-hole recombination that leads to fluorescence [56-58] The band edge transition may not the possible reason for fluorescence as this is the case for typical semiconductors [14]. The experimental data proves that GO shows fluorescence with a lateral range of dimensions. Sun and co-workers has shown fluorescence in the red to NIR region which required lower energy for nanosized GO suspensions [49, 50]. Luo et al has proved that florescence properties of GO suspensions and solid samples are comparable and remain unchanged even with the change in lateral dimensions ranges from 1-10 m [51].This experiment indicates that the lateral size of the sheet is not the main factor for the emission energy. During the reduction reaction of GO with hydrazine vapour by decreasing the intensity the emission peak show redshift towards NIR region [14]. Gokus et al found that a broad red to NIR fluorescence for oxygen-plasma-treated exfoliated graphene [59]. Similar type of fluorescence properties shown by oxygenplasma-treated exfoliated graphene and nano sized synthesized GO indicating that the origin of emission spectra is nearly same [14]. Synthesized GO also exhibit weak blue to UV fluorescence around $390 \mathrm{~nm}$ for thin field and $440 \mathrm{~nm}$ for solution when excited with ultraviolet radiation $[52,54,55]$. Unlike the red to NIR fluorescence here the shift in peaks appears on changing the states from suspension to solid samples due to difference in the dielectric properties of the medium but for the suspended samples further more study is required to understand the effect of environment [52]. The blue fluorescence of quantum dot of graphene is highly $\mathrm{pH}$ dependent, reported by Pan et al [48]. A strong blue fluorescence is observed at the naked eye at a higher $\mathrm{pH}$ and quenched nearly at lower pH ranges. Similar type of blue fluorescence also observed for water soluble GO fragments due to ionic-liquid-assisted electrochemical exfoliation of graphite [60]. The wavelength of the emitted fluorescence is dependent on the size of sp2 clusters. The higher band gaps are observed in sp2 clusters with sizes of less than $1 \mathrm{~nm}$ and upto 20 aromatic rings that give rise to emission in the ultravioletvisible region. Whereas larger sp2 clusters with sizes of more than $2 \mathrm{~nm}$ contain lower gaps give arise red to NIR emission. An alternative explanation for fluorescence in GO is the hydrothermal route to cut GO sheets into blue-luminescent graphene quantum dots, reported by Pan et al.[48]. According to this analysis emission peak from the zigzag site appears due to carbene like free triplet state 11. From the study of previous experiments the fluorescence of functionalized nano material of carbons were attribute to the oxygen containing functional groups but in case of GO enhancement of blue fluorescence indicates that oxygen containing functional groups are may not be the main reason for appearance of fluorescence[52,54,55]. The heterogeneous structure and the sp2 clusters may be the origin for the fluorescence $[52]$. 


\section{Transmission electron microscopy (TEM) in GO}

Data obtain from ultra-high-resolution transmission electron microscopy (TEM) proves that graphene sheet contains sp2 bonded 2D sheet like honeycomb lattice [61]. The structural change occur when graphene is oxidised and GO is formed with an inhomogeneous structure [62]. Three main features comes out- 1 . Holes are found (blue) 2. Graphatic region (yellow) 3. High contrast disordered region (red) The above regions give an idea about high oxidation with a percentage of $2 \%, 16 \%$, and $82 \%$ respectively. The holes are indicating the release of $\mathrm{CO}$ and CO2 during oxidation and exfoliation [63] and graphitic region find out to be less than $5 \mathrm{~nm} 2$. In contrast some of the high resolution electron microscopy studies reported absent of holes in the GO samples [64].

\section{CONCLUSION}

From the detail studies of structure of GO some important information comes out- On the basal plane of
GO it contain some oxygen containing functional groups. Within those functional groups hydroxides and epoxides are present as sp3 hybridised groups. Due to the presence of these groups and a mix environment of sp3 and sp2 hybridised carbons the structure of GO is heterogeneous this leads to the origin of fluorescence and Raman spectra in GO. From the study of TEM it has been proved that GO may contain some holes within the basal plane and those holes are created due to the aggressive oxidation of graphene sheet. From the above summery we may think about the various future aspects 1 . We may try to find out the other oxygen containing functional groups present in the basal plane of GO. 2. From the above data of fluorescence the origin of fluorescence is still unknown. Some group of researcher previously consider the oxygen containing functional groups are responsible for blue fluorescence but recent findings indicates the special arrangement of sp2 clusters within sp3 matrix may be the origin of blue fluorescence. 3. After the detail study of GO we can further proceed to find out the answer of the question is it possible to get back graphene by the reduction of $\mathrm{GO}$ ?
1 Slonczewski JC, Weiss PR. Phys Rev 109,272 (1958).

2 Zhang YB, Tan YW, Stormer HL, Kim P. Nature 438, 201 (2005).

3 Novoselov KS, Jiang Z, Zhang Y, Morozov SV, Stormer HL, Zeitler U, Science 315, 1379 (2007).

${ }^{4}$ Novoselov KS, Geim AK, Morozov SV, Jiang D, Katsnelson MI, Grigorieva IV Nature 438,197 (2005).

5 Cecilia Mattevi, Goki Eda, Stefano Agnoli, Steve Miller, K. Andre Mkhoyan, Ozgur Celik, Daniel Mastrogiovanni, Gaetano Granozzi, Eric Garfunkel, Manish Chhowalla. Nano Lett 8902 (2008)

${ }^{6}$ Lee C, Wei X, Kysar JW, Hone J. Science 321385 (2008)

7 Geim, A. K.; Novoselov, K. S. Nat. Mater. 6183 (2007).

8 Stankovich, S.; Dikin, D. A.; Dommett, G. H. B.; Kohlhaas, K. M.;Zimney, E. J.; Stach, E. A.; Piner, R. D.; Nguyen, S. T.; Ruoff, R. S. Nature 442282 (2006)

${ }^{9}$ X. Li , Y. Zhu, W. Cai, M. Borysiak, B. Han, D. Chen, R. D. Piner ,L. Colombo , R. S. Ruoff, Nano Lett. 94359 (2009)

10 S. Park, R. S. Ruoff, Nat. Nanotechnol. 4217 (2009)

11 Brodie, B. C R. Soc. Lond. A 149249 (1859)

12 Eda, G., Mattevi, C., Yamaguchi, H., Kim, H. and Chhowalla, J. Phys. Chem. C113 15768 (2009)

13 Kian Ping Loh, Qiaoliang Bao, Goki eda and manish chhowalla Nature Chemistry december vol 21015 (2010)

14 C. Hontoria-Lucas, A. J. Lo pez-Peinado, J. d. D. Lo pezGonza lez, M. L. Rojas-Cervantes, R. M. Mart n-Aranda, Carbon 331585 (1995)

15 T. Szabo, E. Tomba cz, E. Ille s, I. De ka ny, Carbon 44 $537(2006)$

16 T. Cassagneau, F. Guerin, J. H. Fendler, Langmuir 167318 (2000)

17 H.-K. Jeong, Y. P. Lee, R. J. W. E. Lahaye, M.-H. Park, K. H. An, I. J. Kim, C.-W. Yang, C. Y. Park, R. S. Ruoff, Y. H. Lee, J. Am. Chem. Soc. 1301362 (2008) .
18 D. W. Boukhvalov, M. I. Katsnelson, J. Am. Chem. Soc. 13010697 (2008)

19 W. Cai, R. D. Piner, F. J. Stadermann, S. Park, M. A. Shaibat, Y. Ishii, D. Yang, A. Velamakanni, S. J. An, M. Stoller, J. An, D. Chen, R. S. Ruoff, Science 3211815 (2008)

20 W. Cai, R. D. Piner, F. J. Stadermann, S. Park, M. A. Shaibat, Y. Ishii, D. Yang, A. Velamakanni, S. J. An, M. Stoller, J. An, D. Chen, R. S. Ruoff, Science 3211815 (2008)

21 H. He, T. Riedl, A. Lerf, J. Klinowski, J. Phys. Chem. 100 19954 (1996)

22 A. Lerf, H. He, T. Riedl, M. Forster, J. Klinowski, Solid State Ionics 101857 (1997)

23 A. Lerf, H. He, M. Forster, J. Klinowski, J. Phys. Chem. B 1024477 (1998)

24 T. Szabo, O. Berkesi, P. Forgo, K. Josepovits, Y. Sanakis, D. Petridis, I. Deka ny, Chem. Mater. 182740 (2006)

25 C. Jian-Hao, W. G. Cullen, C. Jang, M. S. Fuhrer, E. D. Williams, Phys. Rev. Lett. 102236805 (2009)

26 Szabo, T.; Berkesi, O.; Forgo, P.; Josepovits, K.; Sanakis, Y.; Petridis, D.; Dekany, I. Chem. Mater. 182740 (2006)

27 Szabo, T.; Berkesi, O.; Forgo, P.; Josepovits, K.; Sanakis, Y.; Petridis, D.; Dekany, I. Chem. Mater. 182740 (2006)

28 Ishigami, M., Chen, J. H., Cullen, W. G., Fuhrer, M. S. and Williams, E. D Nano Lett. 71643 (2007)

29 Paredes, J. I., Villar-Rodil, S., Sols-Fernndez, P., MartnezAlonso, A. and Tascn, J. M. D. Langmuir 255957 (2009)

${ }^{30}$ Cecilia Mattevi, Goki Eda, Stefano Agnoli, Steve Miller, K. Andre Mkhoyan, Ozgur Celik, Daniel Mastrogiovanni, Gaetano Granozzi, Eric Garfunkel, Manish Chhowalla. Adv. Funct. Mater. 192577 (2009)

31 Kudin, K. et al. Raman spectra of graphite oxide and functionalized graphene sheets. Nano Lett. 836 (2008)

32 Eda, G., Mattevi, C., Yamaguchi, H., Kim, H. and 
Chhowalla, M J. Phys. Chem. 11315768 (2009)

33 Kaiser, A. B., Gmez-Navarro, C., Sundaram, R. S., Burghard, M. and Kern, K. Nano Lett. 91787 (2009) M. Reuter, F. Saueressig, Phys. Rev. D 65 (2002) 065016.

${ }^{34}$ Gmez-Navarro, C. Nano Lett. 101144 (2010)

35 Neil R. Wilson, Priyanka A. Pandey, Richard Beanland, Robert J. Young, Ian A. Kinloch, Lei Gong, Zheng Liu, Kazu Suenaga, Jonathan P. Rourke, Stephen J. York, and Jeremy Sloan. 32547 ACS Nano (2009)

${ }^{36}$ K. A. Mkhoyan, A. W. Contryman, J. Silcox, D. A. Stewart, G. Eda, C. Mattevi, S. Miller, M. Chhowalla, Nano Lett. 91058 (2009)

37 A. Buchsteiner, A. Lerf, J. Pieper, J. Phys. Chem. B 110 22328 (2006)

38 I. Jung, M. Vaupel, M. Pelton, R. Piner, D. A. Dikin, S. Stankovich, J. An, R. S. Ruoff, J. Phys. Chem. C 1128499 (2008)

39 H.-K. Jeong, Y. P. Lee, M. H. Jin, E. S. Kim, J. J. Bae, Y. H. Lee, Chem. Phys. Lett. 470255 (2009)

40 H. C. Schniepp, J. L. Li, M. J. McAllister, H. Sai, M. Herrera-Alonso, D. H. Adamson, R. K. Prudhomme, R. Car, D. A. Saville, I. A. Aksay, J. Phys. Chem. B 1108535 (2006)

41 K. N. Kudin, B. Ozbas, H. C. Schniepp, R. K. Prudhomme, I. A. Aksay, R. Car, Nano Lett. 836 (2007)

42 A. C. Ferrari, J. Robertson, Phys. Rev. B 6114095 (2000)

${ }^{43}$ F. Tuinstra, J. L. Koenig, J. Chem. Phys. 531126 (1970)

44 J. A. Robinson, M. Wetherington, J. L. Tedesco, P. M. Campbell, X. Weng, J. Stitt, M. A. Fanton, E. Frantz, D. Snyder, B. L. VanMil, G. G. Jernigan, R. L. Myers-Ward, C. R. Eddy, D. K. Gaskill, Nano Lett. 92873 (2009)

45 D. C. Elias, R. R. Nair, T. M. G. Mohiuddin, S. V. Morozov, P. Blake, M. P. Halsall, A. C. Ferrari, D. W. Boukhvalov, M. I. Katsnelson, A. K. Geim, K. S. Novoselov, Science 323610 (2009)

46 D. Yang, A. Velamakanni, G. Bozoklu, S. Park, M. Stoller, R. D. Piner, S. Stankovich, I. Jung, D. A. Field, C. A. Ventrice, Jr, R. S. Ruoff, Carbon 47145 (2009)
47 Pan, D., Zhang, J., Li, Z. and Wu, M. Adv. Mater. 22734 (2010)

48 Pan, D., Zhang, J., Li, Z. and Wu, M. Adv. Mater. 22734 (2010)

49 Xiaoming Sun, Zhuang Liu, Kevin Welsher, Joshua Tucker Robinson, Andrew Goodwin, Sasa Zaric, Hongjie Dai Nano Res. 1203 (2008)

${ }^{50}$ Liu, Z., Robinson, J. T., Sun, X. and Dai, H. J. Am. Chem. Soc. 13010876 (2008)

51 Luo, Z. T., Vora, P. M., Mele, E. J., Johnson, A. T. C. and Kikkawa, J. M. Appl. Phys. Lett. 94111909 (2009)

52 Goki Eda, Yun-Yue Lin, Cecilia Mattevi, Hisato Yamaguchi, Hsin-An Chen, I-Sheng Chen, Chun-Wei Chen, Manish Chhowalla 22505 (2009)

53 Tran Viet Cuong, Viet Hung, PhamaQuang, TrungTran, Sung Hong Hahn, Jin SukChung Eun WooShin, Eui JungKim. Mater. Lett. 64399 (2010)

54 Subrahmanyam, K. S., Kumar, P., Nag, A. and Rao, C. N. R. Solid State Commun. 1501774 (2010)

55 Chen, J.-L. and Yan, X.-P. J. Mater. Chem. 204328 (2010)

56 Demichelis, F., Schreiter, S. and Tagliaferro, A. Phys. Rev. B 512143 (1995)

57 Rusli, Robertson, J. and Amaratunga, G. A. J. J. Appl. Phys. 802998 (1996)

58 Koos, M., Veres, M., Fule, M. and Pocsik, I. 1153 (2002)

59 Gokus, T. et al. ACS Nano. 33963 (2009)

60 Jiong Lu, Jia-xiang Yang, Junzhong Wang, Ailian Lim, Shuai Wang, and Kian Ping Loh ACS Nano 32367 (2009)

61 J. C. Meyer, A. K. Geim , M. I. Katsnelson , K. S. Novoselov , D. Obergfell , S. Roth , C. Girit , A. Zettl , Solid State Commun. 143101 (2007)

62 Kris Erickson, Rolf Erni , Zonghoon Lee, Nasim Alem , Will Gannett, and Alex Zettl Adv. Mater. 224467 (2010)

63 J. T. Paci, T. Belytschko, G. C. Schatz, J. Phys. Chem. C 11118099 (2007)

${ }^{64}$ R. S. Pantelic, J. C. Meyer , U. Kaiser , W. Baumeister, J. M. Plitzko , J. Struct. Biol. 170152 (2010) 